\title{
DIVISION AND EXTENSION IN WEIGHTED BERGMAN-SOBOLEV SPACES
}

\author{
JOAQUín M. ORTEGa a.to JOAN Fábriega
}

Abstract

Let $D$ he a bounded strictly psendoconvex domain of $C^{n t}$ with co boumtiny and $Y=\{z ; u(z)=\cdots=u(z)=0\}$ is holomorplice submanifold in an neighbourhood of $\bar{D}$, of coclimension $t$ and transwersal to the bonindary of $D$.

In this work we give al decomposition formula $f=u_{1} f_{1}+\cdots+$ $u_{t} f_{l}$ for functions $f$ of the Bergman-Sobolev space vanishing on $M=Y \cap D$. Also we givo nocessary and sufficient conclitions on it set of holomorphic functions $\left\{f_{0}\right\}_{|a| \leq m}$ on $M$, so that there cxists a holomorphic function in the Bergman-Sobolev spawe such that $\left.D^{\alpha} f\right|_{M}=f_{\alpha}$ for atl $|\alpha| \leq m$.

\section{Introduction and main results}

Let $D=\{z ; \rho(z)<0\}$ be a bounder strictly psendoconvex domain of $C^{n}$ with $\mathcal{C}^{\infty}$-boundary. Let $Y=\left\{z ; u_{1}(z)=\ldots=u_{l}(z)=0\right\}$ denote a holomorphic; submanifold in is neighbourhood of $\bar{D}$, of codimension $l$ and transversal to the boundary of $D \cap Y$, i.e. $\partial \rho \wedge \partial u_{1} \wedge \ldots \wedge \partial u_{i} \neq 0$ on the intersection of the boundary of $D$ and the submanifold $Y$.

For every $1 \leq p<\infty, \delta>0$, and $k=0,1, \ldots$ we consider the weighted Sobolev space

$$
L_{\delta, k}^{p}(D)=\left\{f \text { moasurable }:\|f\|_{p, \delta, k}<\infty\right\}
$$

where

$$
\|f\|_{\gamma, \delta, k}=\sup \left\{\left(\int_{D}\left|D^{\alpha} \bar{D}^{\beta} f\right|^{p}(-\rho)^{\delta-1}\right)^{\frac{1}{\prime \prime}} ;|\alpha|+|\beta| \leq k\right\}
$$

\footnotetext{
Partially supported by the grant. PB89-0311 of the DGICYT. Spain.
} 
and $D_{z}^{\alpha}=\frac{\partial^{|\alpha|}}{\partial z_{\alpha}}, \bar{D}_{z}^{\beta}=\frac{\partial^{|; \beta|}}{\partial \bar{z}_{\beta}}$

Also, we define for cvery $p, \delta, k$ the weighted Bergman-Sobolev space as the space of holomorphic functions $A_{\delta_{1} k}^{p}(D)=L_{\delta, k}^{p}(D) \cap \mathcal{O}(D)$.

Replacing the derivatives $D_{z}^{\alpha}, \bar{D}_{z}^{\beta}$ for tangent-derivatives on the submanifold $Y$, we define in the same way the spaces $L_{\delta, k}^{p}(M)$, and $A_{\delta, k}^{p}(M)$ in the submanifold $M=Y \cap D$.

It is well known (sce for instance $[3],[\mathbf{4}]$ ) that

$$
A_{\delta, k}^{p}(D) \subset A^{t}(D), \text { if } t=k-\frac{n+\delta}{p}>0
$$

where $A^{t}(D)$ denotes the corresponding space of the holomorphic Lipschitz functions. It is also well known that

$$
A_{\delta, k}^{p}(D)=A_{\delta^{\prime}, k^{\prime}}^{p^{\prime}}(D), \quad \text { if } \delta-\delta^{\prime}=p\left(k-k^{\prime}\right) .
$$

One of the main results that we will prove in this work is a result of division in the spaces $A_{\delta, k}^{p}(D)$.

We recall the following result of division in the holomorphic Lipschitz spaces, due to P. Bonneau, A. Cumenge and A. Zériahi ([6]):

If $f$ is a holomorphic Lipschitz function of class $A^{t}(D)$ vanishing in the submanifold $M$, then there exist functions $f_{j}, j=1, \ldots, l$. of class $A^{t-\frac{1}{2}}(D)$ such that $f=u_{1} f_{1}+\ldots+u_{l} f_{l}$.

We prove in this paper the following theorem:

Theorem 1.1.

If $f$ is a function of class $A_{\delta, k}^{p}(D)$ vanishing on the submanifold $M=Y \cap D$ transversal to the boundary of $D$, then there exist functions $f_{j}, j=1, \ldots$, l of class $A_{\delta+\frac{p}{2}, k}^{p}(D)$ such that

$$
f=\sum_{j=1}^{l} u_{j} f_{j} .
$$

Observe that by (1.1) and (1.2) the Theorem 1.1 is in some sense a refinement of the above result of division in the holomorphic lipschitz spaces.

In the limit case where $Y$ is a point $\zeta$ of $D$, the Theorem 1.1 is the Gleason's problem. In this case (see $[\mathbf{1 1}]$ ) it is known that

$$
f(z)=\sum_{j=1}^{n}\left(z_{j}-\zeta_{j}\right) f_{j}(z), \quad f_{j} \in A_{\delta, k}^{p}(D) .
$$


The second main result that we will prove is an extension theorem of jets. This consists to give necessary and sufficient conditions on a set $\left\{f_{\alpha}\right\}_{|\alpha x| \leq m}$ ol holomorphic functions on the submanifold $M=Y \sqcap D$ so that there exists a $A_{\delta, k}^{p}(D)$-function $f$, such that $\left.D_{z}^{\alpha} f\right|_{M}=f_{\alpha}$ for all $[\alpha] \leq n$.

The case $m=0$, i.e. the problem of extension and restriction of functions of class $A_{\delta, k}^{p}(D)$, has been studied by many authors using different methods. (See for exemple $[\mathbf{3}],[\mathbf{4}],[9]$ ). The result oblained in this case is

$$
\left.A_{\delta, k}^{p}(D)\right|_{M}=A_{\delta+1, k}^{p}(M) .
$$

The above problem in the holomorphic Lipschitz spaces has been proved by us in $[\mathbf{1 2}]$.

In order to state the result of extension let us introduce the following definitions.

We consider smoolh vector fields on $\bar{D}$

$$
X=\sum_{i=1}^{n} a_{i}(z) \frac{\partial}{\partial z_{i}}
$$

For these vector fields we say that; $X$ is complex-tangential if $X \rho(z)=$ 0 for every $z$ in a neighbourhood of the boundary of $D$, and we define its weight $w(X)$ in the usual way:

$$
w(X)= \begin{cases}\frac{1}{2} & \text { if } X \text { is complex-tangential } \\ 1 & \text { in other case. }\end{cases}
$$

If $X=X_{k} \ldots X_{1}$ is a differential operator we define its weight by

$$
w(X)=\sum_{i=1}^{k} w\left(X_{i}\right)
$$

We recall that for a holomorphic function $f$ on $D$ the $j-t h$ covariant differential of $f$ at a point $z \in D$ is defined by:

$$
\begin{aligned}
d^{0} f_{z} & =f(z) \\
d^{j} f_{z}\left(X_{1}, \ldots, X_{j}\right) & =X_{j} d^{j-1} f_{z}\left(X_{1}, \ldots, X_{j-1}\right)- \\
& \sum_{i=1}^{j-1} d^{j-1} f_{z}\left(X_{1}, \ldots, \nabla_{X_{j}} X_{i}, \ldots, X_{j-1}\right)
\end{aligned}
$$


and that in coordinates we can write

$$
d^{j} f_{z}=\sum_{|j|=j} \frac{\partial^{j} f(z)}{\partial \zeta_{i_{1}} \ldots \partial \zeta_{i_{j}}} d z_{i_{1}} \otimes \ldots \otimes d z_{i_{j}}
$$

Also, fixed $m$, we denote by

$$
J_{n n} f_{z}=\left(d^{0} f_{z}, \ldots, d^{r n} f_{z}\right)
$$

the holomorphic jet of order $m$ at the poinl $z \in D$ induced by $f$.

Morcover, if the function $f$ is of class $A_{\delta, k}^{p}(D)$, then it is well known (see $[1],[3],[4])$ that the function $\left.d^{j} f_{z}\left(X_{1}, \ldots, X_{j}\right)\right|_{M}$ is of class $L_{\delta+t+w(X) p, k}^{p}(M)$ where $X$ is the differential operator formed by the vector fields $X_{1}, \ldots, X_{j}$.

Thus, if we define the covariant tensors of order $j$ at a point $z \in M$ as

$$
F_{z}^{j}=d^{j} f_{z}
$$

then they satisly the following conditions for every $0 \leq j \leq m$ :

I-1) At every point $z \in M, F_{z}^{j}$ is a $j$-covariant symmetric tensor.

I-2) $F^{j}\left(\frac{\partial}{\partial z_{1}}, \ldots, \frac{\partial}{\partial z_{1}}, \ldots, \frac{\partial}{\partial z_{n}}, \ldots, \frac{\partial}{\partial z_{n}}\right)$ are holomorphic functions on $\mathrm{M}$.

I-3) $F^{j}\left(X_{1}, \ldots, X_{j}\right)=X_{j} F^{j-1}\left(X_{1}, \ldots, X_{j-1}\right)-$

$$
\sum_{i=1}^{j-1} F^{j-1}\left(X_{1}, \ldots, \nabla_{X_{j}} X_{i}, \ldots, X_{j-1}\right)
$$

for every tangent vector field $X_{j}$ at $\mathrm{M}$.

I-4) $F^{j}\left(X_{1}, \ldots, X_{j}\right) \in L_{\delta+1+w(X) p_{i}, k}^{p}(M)$.

Therefore, it is natural to introduce the following definition:

Definition 1.2.

$F=\left(F^{0}, \ldots, F^{m}\right)$ is an $A_{\delta, k}^{p}$-jet of order $m$ on $\mathrm{M}$ if it satisfics the four previous conditions.

The condition I-3) just gives a relation of coherence between the tensors $F^{j}$. We point out that a $A_{\delta_{i} k}^{p}$-jet on $M$ of order 0 is a function of class $A_{\delta+l, k}^{p}(M)$.

The notation of $A_{\delta, k}^{P}$-jet is justified by the following result. 


\section{Theorem 1.3.}

$F=\left(F^{0}, \ldots, F^{m n}\right)$ is a $A_{\delta, k}^{p}$-jet of order $m$ on $M$ if and only if there exists a function $f$ of class $A_{\delta, k}^{p}(D)$ such that $j_{n} f=F$ on $M$.

We recall that in $[12]$ we said that $F^{\prime}=\left(F^{0}, \ldots, F^{m}\right)$ is an $A^{t}$-jet if it satisfies the conditions I-L, I-2, I-3 of the Definition 1.2 and the condition

$$
\left|X_{k} \ldots X_{j+1} F^{j}\left(X_{1}, \ldots, X_{j}\right)\right| \leq c M(t-w(X), z)
$$

where the function $M(s, z)$ is defined by

$$
M(s, z)= \begin{cases}1 & \text { if } s>0 \\ |\log | \rho(z)|| & \text { if } s=0 \\ |\rho(z)|^{s} & \text { if } s<0\end{cases}
$$

and the vector fields $X_{j+1}, \ldots, X_{k}$ are tangential to the subrnanifold $Y$.

In the same paper [12] we proved that:

(1.6) $F$ is a $A^{t}$-jet of order $m$ on $M$ if and only if there exists a holomorphic Lipschitz function $f$ of class $A^{t}(D)$ such that $J_{m} f=F$ on $M$.

To prove the Theorem 1.3 we will use the Theorem 1.1, the results (1.1), (1.2) and (1.6) and a result of resolution of the $\vec{\partial}$-equation in the spaces $L_{b, k}^{p}(D)$.

As usually several diferent constants in the inequalities will be denoted by $c$.

\section{Some integral formulas}

In this section we give an extension operator and an explicit, solution of the $\bar{\partial}$-equation.

We denote by $\Phi(\zeta, z)$ the support function of Fenkin and we put $a(\zeta, z)=-\rho(\zeta)+\Phi(\zeta, z)$.

Using the results of $B$. Berndtsson and $M$. Andersson [5], Cor every positive integer ss we can construct kerncls $K^{s}$ and $R^{s}$ of type

$$
\begin{aligned}
K^{s}(\zeta, z) & =\left(\frac{-\rho(\zeta)}{a(\zeta, z)}\right)^{n+s} \frac{\varphi_{0}(\zeta, z)}{|\zeta-z|^{2 n}}+ \\
& \sum_{j=1}^{n-1} \frac{\left(-\rho(\zeta)^{n+s-j} \varphi_{j}(\zeta, z)\right.}{a(\zeta, z)^{n+s+1}|\zeta-z|^{2 n-2 j}} \\
R^{s}(\zeta, z) & =\frac{(-\rho(\zeta))^{s} \varphi_{n}(\zeta, z)}{a(\zeta, z)^{n+s+1}}
\end{aligned}
$$


which have the following properties:

1. $d_{\zeta, z} K^{s}=R^{s}$ outside the diagonal, and $R^{s}$ is holomorphic in the variable $z$.

2. The forms $\varphi_{j}, j=0, \ldots, n$ are of class $\mathcal{C}^{\infty}(\bar{D} \times \bar{D})$.

3. $\left|\varphi_{j}(\zeta, z)\right| \leq c|\zeta-z|, \quad j=0, \ldots, n-1$.

4. Koppelman Formulas. Let $K_{p, q}^{s}$ be the component of $K^{s}$ of bidegree $(p, q)$ in $z,(n-p, n-q-1)$ in $\zeta$, and let $R_{p, q}^{s}$ be the component of $R^{s}$ of bidegree $(p, q)$ in $z$, and $(n-p, n-q)$ in $\zeta$. Then, if $f$ is $a(p, q)$ form with coefficients in $\mathcal{C}^{1}(\bar{D})$, we have

$$
\begin{gathered}
f(z)=(-1)^{p+q+1} \int_{D} \bar{\partial} f(\zeta) \wedge K_{p, q}^{s}(\zeta, z)+ \\
(-1)^{p+q} \bar{\partial}_{z} \int_{D} f(\zeta) \wedge K_{p, q-1}^{s}(\zeta, z), \quad \text { if } q \geq 1 \\
f(z)=(-1)^{p+1} \int_{D} \bar{\partial} f(\zeta) \wedge K_{p, 0}^{s}(\zeta, z)- \\
\int_{D} f(\zeta) R_{p, 0}^{s}(\zeta, z), \quad \text { if } q=0 .
\end{gathered}
$$

Now, if $Y=\left\{z_{i} z_{1}=\ldots=z_{i}=0\right\}$ and $M=Y \cap D$, then the same construction used in [5] to prove these results gives for each $s>\frac{\delta-1}{p}$ an extension operator from the space $A_{\delta, k}^{p}(M)$ to the space of holomorphic functions $\mathcal{O}(D)$. This operator is defined by

$$
E^{s} f(z)=\int_{M} f(\zeta) R_{M}^{s}(\zeta, z)
$$

where

$$
R_{M}^{s}(\zeta, z)=\frac{(-\rho(\zeta))^{s}}{a(\zeta, z)^{n-l+1+s}} \varphi(\zeta, z), \quad \zeta \in M, z \in D
$$

and the form $\varphi$ has coefficients of class $\mathcal{C}^{\infty}(\bar{M} \times \bar{D})$ and it is holomorphic in $z$.

Moreover, the same formula (2.3) also gives an explicit integral operator to solve the $\vec{\partial}$-equation for $(0, q)$ forms $\bar{\partial}$-closed. This operator is given by the kernel $K_{0 ; q-1}^{s}(\zeta, z)$.

The estimates for these kcrnels are given by the following Lemma. 


\section{Lemma 2.1.}

Lel $j \leq 2 n-1$ be an integer. Then with $M(s, z)$ defined as (1.5) we have

$$
\int_{D} \frac{1}{|a|^{t}|\zeta-z|^{j}} \leq c \begin{cases}M\left(n+1-t-\frac{j}{2}, z\right) & \text { if } j \leq 2 n-3 . \\ 1 & \text { if } j=2 n-2, t<2 . \\ M(2-t, z)|\log | \rho(z)|| & \text { if } j=2 n-2, t \geq 2 . \\ M(1-t, z) & \text { if } j=2 n-1 .\end{cases}
$$

Proof:

Using the usual change of coordinates and computing the respective integrals we obtain these estimates. (Sec for instance [10]).

Now we will state some formulas of integration by parts.

The first formula is contained in the following Lemma of $\left[7_{]}\right.$:

\section{Lemma 2.2.}

Let $f$ be a $(0,1)$ form $\bar{\partial}$-closed with coefficients of class $\mathcal{C}^{1}(\bar{D})$. Then

$$
D_{z}^{\alpha} g=-\int_{D} D_{\zeta}^{\circ} \int \wedge K_{0,0}^{s}+\sum \int_{D} D_{\zeta}^{\gamma} \int \wedge D_{z}^{\beta} R_{0,1,1}^{s, i}
$$

where in the last terms $\gamma$ and $\beta$ are mulliindexes with $|\gamma|+|\beta|=|\alpha|-1$, $i=1, \ldots, n$, and $R_{0,1,1}^{s, i}$ denotes the coefficient of $d z_{i}$ in the component of the kernel $R^{s}$ of degree $(1,0)$ in $z$ and $(n, n-1)$ in $\zeta$.

Before to state the second formula we introduce the following kernels, that are a generalization of the extension kernels $R_{M}^{s}$.

\section{Definition 2.3.}

If $Y=\left\{z_{1}=\ldots=z_{l}=0\right\}$ and $M=Y \cap D$, wc define the kernels

$$
R_{M, \psi}^{s, r^{r}}(\zeta, z)=(-\rho(\zeta))^{s} \psi(\zeta, z), \quad \zeta \in M, z \in D
$$

where the form $\psi(\zeta, z)$ has the coefficients of class $\mathcal{C}^{\infty}(M \times D)$, and it satisfies

$$
\left|D_{z}^{\alpha} D_{\zeta}^{\beta} \bar{D}_{\zeta}^{\gamma} \psi(\zeta, z)\right| \leq c|a(\zeta, z)|^{r-\left(|\alpha|+\left|\frac{|\beta|}{2}+\right| \gamma \mid\right)}
$$

for every multindexes $\alpha, \beta, \gamma$.

Let also $R_{M, \psi}^{p, r}$ denote the integral operator given by this kernel.

Observe that the externsion operator $R_{M}^{s}(\zeta, z)$ is a $R_{\psi, M}^{s,-(n-t+1+s)}$ operator, because $\left|D_{\zeta} a(\zeta, z)\right| \leq c|\zeta-z| \leq c|a(\zeta, z)|^{\frac{1}{2}}$.

These operators have the following properties: 
Lemma 2.4 .
i) $D_{z}^{\alpha} R_{M, \psi}^{s, r}=R_{M, \psi_{1}}^{s, r-|\alpha|}$
ii) $\int_{M}\left|R_{M, \psi}^{s, r}\right| \leq c M(n-l+1+s+r, z)$.

Proof:

i) is clear and ii) follows from Lemma 2.1.

\section{Lemma 2.5.}

If $f$ is a function of class $\mathcal{C}^{k}(\bar{M})$, then, fixed an integer $q$, we can find operators $R_{M, \psi_{\gamma}}^{s_{\gamma}, F_{\gamma}}, R_{M_{i} \psi_{\mu}}^{s_{\mu}, T_{\mu}}$ such that

$$
D_{z}^{\alpha} R_{M, \psi}^{s, r} f=\sum_{\substack{|\gamma|=k \\ s_{\gamma}+r_{\gamma} \geq s+r+k-|\alpha| \\ p_{\gamma} \geq s+k}} R_{M, \psi_{\gamma}}^{s_{\gamma}, r_{\gamma}} D_{\zeta}^{\gamma} f+
$$

Remark. Roughly speaking, the Lemma 2.4 prove that the coefficient $s+r$ measures the regularity of the operator $R_{M, \psi}^{s, r}$, and therefore the operators $R_{M_{,}, \psi_{\gamma}}^{s_{\gamma}, r_{\gamma}}$ in Lemma 2.5 have at least the same regularity than the operator $R_{M, \psi}^{s, r}$ plus $k-|\alpha|$. On the other hand, choosing $q$ large enought we can assume that the operators $R_{M, \psi_{j k}}^{s_{\mu} r_{\mu}}$ are as regular as required.

Proof:

Using the transversavility of the submanifold $Y$, we can choose a covering $\left\{U_{i}\right\}_{i=0}^{i_{0}}$ of $M$ such that

i) $M=\cup_{i=0}^{i_{0}} U_{i}$, and $U_{0}=\{z: \rho(z)<-\delta\}, \delta>0$.

ii) For each $i, 1 \leq i \leq i_{0}$ there is $l+1 \leq j_{i} \leq n$ such that $\frac{\partial \rho(z)}{\partial z_{j_{i}}} \neq 0$ on $U_{i}$.

Let $\left\{\chi_{i}\right\}$ be a partition of the unity for this covering and we put

$$
R_{M, \psi}^{s_{i} r}=\sum_{i=0}^{i_{0}} R_{M, \chi_{i} \psi}^{s, r} .
$$

We want to prove the Lemma for each one of the operators of the sum.

If $i=0$ the result is clear by the properties of $\psi$ and the property i) of the covering. 
If $i \geq 1$ by the property ii) of the covering we have

$$
\begin{gathered}
\int_{M} R_{M, \psi, \psi}^{s, r}(\zeta, z) f(\zeta)= \\
\frac{1}{s+1} \int_{M}(-\rho(\zeta))^{s+1} \frac{\partial}{\partial \zeta_{j_{i}}}\left(\chi_{i} \psi(\zeta, z)\left(\frac{\partial \rho(\zeta)}{\partial \zeta_{j_{i}}}\right)^{-1} f(\zeta)\right)= \\
\int_{M} R_{M, \chi_{i} \psi^{\prime}}^{s+1, r} \frac{\partial f}{\partial \zeta_{j_{i}}}+\int_{M} R_{M, \chi_{i} \psi^{\prime \prime}}^{s+1, r-\frac{1}{2}} f
\end{gathered}
$$

Iterating this process in the terms which have less than $k$ derivatives on the function $f$, and using the Lemma 2.4 i) we obtain the result.

\section{Solution of the $\bar{\partial}$-equation in the $L_{\delta, k}^{p}(D)$ space}

The airn of this section is to prove the following Theorems.

\section{Theorem 3.1.}

If $f$ is a $(0, q)$ form $\bar{\partial}$-closed with coefficients of class $L_{\delta, 0}^{p}(D), 1 \leq p<$ $\infty, \delta>0$, then there exists a $(0,1-1)$ form $g$ with coefficients of class $L_{\delta^{*}, 0}^{p}(D)$ for all $\delta^{*} \geq \delta-\frac{p}{2}, \delta^{*}>0$ such that $\bar{\partial} g=f$.

\section{Theorem 3.2.}

If $f$ is a $(0,1)$ form $\bar{\partial}$-closed with coefficients of class $L_{\delta, k}^{p}(D), 1 \leq p<$ $\infty, \delta>0, k=0,1, \ldots$, then there exists a function $g$ with coefficients of class $L_{\delta^{*}, k}^{p}(D)$ for all $\delta^{*} \geq \delta-\frac{p}{2}, \delta^{*}>0$ such that $\bar{\partial} g=f$.

To prove these Theorems we need the following Lemma.

\section{Lemma 3.3.}

If a kernel $K(\zeta, z)$ satisfies $|K(\zeta, z)| \leq c \frac{\left(-p(\zeta)^{s}\right.}{|a(\zeta, z)|^{2}|\zeta-z|^{z}} s, t \geq 0: j=$ $0, \ldots, 2 n-1$, and $f$ is of class $L_{\delta, 0}^{p}(D), 1 \leq p<\infty, 0<\delta-1<s p$, then the function $K f$ is of class $L_{\delta^{*}, 0}^{p}(D), \delta^{*} \geq \delta-\lambda p, \delta^{*}>0$, where

$$
\lambda= \begin{cases}n+1+s-t-\frac{j}{2} & \text { if } j \leq 2 n-2 \\ 2-\varepsilon+s-t & \text { if } j=2 n-2, \varepsilon>0 \\ 1+s-t & \text { if } j=2 n-1 .\end{cases}
$$




\section{Proof:}

We want to see that for a $\delta^{*}$ fixed which satisfies the previous conditions we have

$$
\begin{aligned}
I= & \int_{D}\left(\int_{D}|K(\zeta, z)| f(\zeta) \mid d \zeta\right)^{p}(-\rho(z))^{\delta^{*}-1} d z \leq \\
& c \int_{D}|f(\zeta)|^{p}(-\rho(\zeta))^{\delta-1} d \zeta .
\end{aligned}
$$

First we consider the case $p=1$ and $j \neq 2 n-2$.

In this case applying the Fubini Theorem we have

$$
I \leq c \int_{D}|f(\zeta)|(-\rho(\zeta))^{s} \int_{D} \frac{(-\rho(z))^{\delta^{*}-1}}{|a(\zeta ; z)|^{t}|\zeta-z|^{j}} d z d \zeta
$$

and using that $|a(\zeta, z)| \approx|a(z, \zeta)|,-\rho(z) \leq c|a(\zeta, z)|$ and the Lemma 2.1 we get

$$
I \leq c \int_{D}|f(\zeta)|(-\rho(\zeta))^{s} M\left(\delta^{*}-1+\lambda-s, \zeta\right) d \zeta .
$$

Now, if $\delta^{*}-1+\lambda-s \geq 0$ we have that $(-\rho(\zeta))^{s} M\left(\delta^{*}-1+\lambda-s, \zeta\right) \leq$ $c(-\rho(\zeta))^{\delta-1}$, since $s>\delta-1$.

Moreover, if $\delta^{*}-1+\lambda-s<0$ then $(-\rho(\zeta))^{s} M\left(\delta^{*}-1+\lambda-s, \zeta\right) \leq$ $c(-\rho(\zeta))^{\delta^{*}-1+\lambda} \leq c(-\rho(\zeta))^{\delta-1}$ because $\delta^{*} \geq \delta-\lambda$.

Hence

$$
I \leq c \int_{D}|f(\zeta)|(-\rho(\zeta))^{\delta-1} d \zeta
$$

If $p=1$ and $j=2 n-2$ we obtain in (3.1) the estimate

$$
I \leq c \int_{D}|f(\zeta)|(-\rho(\zeta))^{s} M\left(\delta^{*}+1-t, \zeta\right)|\log | \rho(\zeta) \mid d \delta
$$

and applying the same reasoning as in the above case we prove the result.

Now we consider the case $1<p<\infty$ and $j \leq 2 n-3$.

Let $p^{\prime}=\frac{p}{p-1}$. Taking $r$ such that

$$
\frac{p-1}{p}\left(n+1-\frac{j}{2}\right)<r<\frac{p-1}{p}\left(n+1-\frac{j}{2}+\frac{\delta^{*}}{p-1}\right)
$$


and applying the Hölder inequalities we get

$$
\begin{aligned}
I & \leq c \int_{D}\left(\int_{D}|f(\zeta)|^{p} \frac{(-\rho(\zeta))^{s p p}}{|a(\zeta, z)|^{(t+r) p}|\zeta-z|^{j}} d \zeta\right) \\
& \left(\int_{D} \frac{1}{|a(\zeta, z)|^{p p^{\prime}}|\zeta-z|^{j}} d \zeta\right)^{\frac{p}{p^{\prime}}}(-\rho(z))^{\delta^{*}-1} d z \leq \\
& \leq c \int_{D} \int_{D}|f(\zeta)|^{p}(-\rho(\zeta))^{s p} \frac{\left(-\rho(z)^{\left(n+1-r p^{\prime}-\frac{j}{2}\right)(p-1)+\delta^{*}-1}\right.}{|a(\zeta, z)|^{(t-r) p}|\zeta-z|^{j}} d \zeta d z .
\end{aligned}
$$

By Fubiui Theorem and the Lemma 4.2 we have

$$
\begin{aligned}
I \leq & c \int_{D}|f(\zeta)|^{p}(-\rho(\zeta))^{s p} M\left(n+1-(l-r) p-\frac{j}{2}+\right. \\
\left.\left(n+1-r p^{\prime}-\frac{j}{2}\right)(p-1)+\delta^{*}-1, \zeta\right) d \zeta= & \\
& c \int_{D}|f(\zeta)|^{p}(-\rho(\zeta))^{s p} M\left(\left(n+1-t-\frac{j}{2}\right) p+\delta^{*}-1, \zeta\right) d \zeta \leq \\
& c \int_{D}|f(\zeta)|^{p}(-\rho(\zeta))^{\delta-1} d \zeta
\end{aligned}
$$

and hence this case is proved.

The cases $1<p<\infty$ and $j=2 n-2,2 n-1$ follow in the same way laking $r$ such that

$$
\frac{p-1}{p}(2 n-j)<r<\frac{p-1}{p}\left(2 n-j+\frac{\delta^{*}}{p-1}\right)
$$

\section{Corollary 3.4.}

If $R_{D_{1} \psi}^{s, r}$ is the operator of the Definition 2.3 and $f$ is of class $L_{\delta, k}^{p}(D)$, $\delta-1<s p$, then the function $R_{D, \psi}^{s, r} f$ is of class $L_{\delta^{*}, k}^{p}(D)$, for all $\delta^{*} \geq$ $\delta-(n+1+s+r) p, \delta^{=}>0$.

\section{Proof:}

Applying the Lemmas $2.5,3.3$ we obtain the result.

Proof of Theorem 3.1:

We take $s>0$ such that $s p>\delta-1$ and we define the function $g=-\int_{D} f \wedge K_{0,4-1}^{s}$, where the kernel $K^{s}$ is given in (2.1).

It is clear by (2.3) that $\bar{\partial}_{g}=f$. Now, using the estimate

$$
\left|K^{s}\right| \leq c\left(\frac{(-\rho)^{n+s}}{|a|^{n+s}|\zeta-z|^{2 n-1}}+\sum_{i=1}^{n-1} \frac{(-\rho)^{n+s-i}}{|a|^{n+1+s}|\zeta-z|^{2 n-2 i-1}}\right)
$$


and applying the Lemma 3.3 we obtain the result.

Proof of Theorem 3.2:

We define $g$ as in the previous Theorem.

By Lemmas 2.2 and 2.4 we have

$$
D_{z}^{\alpha} g=-\int_{D} D_{\zeta}^{\alpha} f \wedge K_{0,0}^{s}+\sum_{|\gamma|+|\beta|<|\alpha|} \int_{D} D_{\zeta}^{\gamma} f \wedge R_{D, \psi_{\gamma}}^{s,-(n+1+s+|\beta|)}
$$

where the kernels $R_{D, \psi_{\gamma}}^{s,-(n+1+s+|\beta|)}$ are holomorphic in $z$.

The same reasoning used in the proof of Theorem 3.1 shows that the term $\int_{D} D_{\zeta} f \wedge K_{0,0}^{s}$ is of class $L_{\delta^{*}, 0^{*}}^{p}$

Moreover the Corollary 3.4 shows that the term

$$
\int_{D} D_{\zeta}^{\gamma} f \wedge K^{s_{1}-(n+1+s+|\beta|)}
$$

is of class $A_{\delta+|\beta| p, k-|\gamma|}^{p}(D)=A_{\delta,|\alpha|-|\beta|-|\gamma|}^{p}(D)$.

Now, using that $|\alpha|-|\beta|-|\gamma| \geq 1$ we end the proof.

\section{Division in the $A_{\delta, k}^{p}$ spaces}

To prove the Theorem 1.1, we will first solve the problem locally using the following projection.

\section{Lemma 4.1.}

Let $Y=\left\{z ; z_{1}=\ldots=z_{l}=0\right\}$ be a linear submatifold transversal to the boundary of $D$. Then for every point $w$ in the boundary of $M=Y \cap D$, there exists a neighbourhood $V$ of $w$ and a projection

$$
\Pi: V \longrightarrow V \cap Y
$$

of class $\mathcal{C}^{\infty}(\bar{V})$, such that

i) $\Pi(z)=z+z_{1} g_{1}+\ldots+z_{i} g_{t}$

ii) $\rho(\Pi(z)) \leq \rho(z)-c\left|z^{\prime}\right|^{2}, \quad z^{\prime}=\left(z_{1}, \ldots, z_{l}, 0 \ldots, 0\right), c>0$

iii) $|a(\zeta, z)| \leq c \mid a\left(\zeta, \operatorname{ll}(z) \mid \leq c\left(|a(\zeta, z)|+\left|z^{\prime}\right|^{2}\right)\right.$

Remark. Observe that the condition ii) implies that if $z \in V \cap D$ then $\Pi(z) \in V \cap M$. 
Proof:

We write

$$
\begin{gathered}
\left\langle\zeta_{1} z\right\rangle=\sum_{i=1}^{\pi} \zeta_{i} z_{i} \quad, \quad z^{\prime \prime}=z-z^{\prime} \\
\frac{\partial \rho}{\partial \zeta}=\left(\frac{\partial \rho}{\partial \zeta_{1}}, \ldots, \frac{\partial \rho}{\partial \zeta_{n}}\right), \quad \frac{\partial \rho}{\partial \zeta^{\prime \prime}}=\left(0, \ldots, 0, \frac{\partial \rho}{\partial \zeta_{l+1}}, \ldots, \frac{\partial \rho}{\partial \zeta_{n}}\right) .
\end{gathered}
$$

Let $U$ be a neighbourhood of the boundary of $M$. Shrinking $U$ and using the transversavility of $Y$ we can assume that $\left|\frac{\partial \rho}{\partial z^{\prime \prime}}\right| \geq c>0$ on $U$ and therefore, for every $1 \leq j \leq l$, we can take a function $h^{j}: U \longrightarrow C^{n}$ of class $\mathcal{C}^{\infty}(U)$ such that

$$
h^{j}=\left(0, \ldots,-1_{j}, \ldots, 0, h_{l+1}^{j}, \ldots, h_{r}^{j}\right), \text { and }\left\langle\frac{\partial \rho}{\partial z}, h^{j}\right\rangle=0 .
$$

The next step is to see that for a certain $d>0$ the projection

$$
\Pi(z)=z+z_{1} h^{1}+\ldots+z_{l} h^{t}-d\left|z^{\prime}\right|^{2} \frac{\partial \rho(z)}{\partial z^{\prime \prime}}
$$

satisfies the required conditions.

It is obvious that II satisfics i) for every $d$.

Using the Taylor development and the properties (4.1) we have that

$$
\begin{gathered}
\rho(\Pi(z)) \leq \rho(z)-2 d\left|z^{\prime}\right|^{2}\left|\frac{\partial \rho}{\partial z^{\prime \prime}}\right|+c_{0}|\operatorname{II}(z)-z|^{2} \leq \\
\rho(z)-\left(2 d c_{1}-c_{2}\right)\left|z^{\prime}\right|^{2}+c_{3} d\left|z^{\prime}\right|^{3}
\end{gathered}
$$

where $c_{1}, c_{2}, c_{3}>0$.

Now taking $d$ such that $2 d c_{1}-c_{2}>c>0$ and shrinking $U$ we obtain ii).

lo prove iii) we recall that $\Phi(\zeta, z)$ is holomorphic in $z$ and

$$
\Phi(\zeta, z)=\langle P(\zeta, z), \zeta-z\rangle=\left\langle\frac{\partial \rho}{\partial \zeta}, \zeta-z\right\rangle+O\left(|\zeta-z|^{2}\right) .
$$

Using this and the properies (4.1), we have

$$
\begin{aligned}
a(\zeta, z)-a(\zeta, \Pi(z))= & \langle P(\zeta, z)-P(\zeta, \Pi(z)), \zeta-z\rangle+ \\
& \langle P(\zeta, \Pi(z)), z-\Pi(z)\rangle=\sum_{j=1}^{l} z_{j} \psi(\zeta, z)
\end{aligned}
$$

with

$$
|\psi(\zeta, z)| \leq c\left(|\zeta-z|+\left|z^{\prime}\right|\right) \approx c\left(|\zeta-\mathrm{\Pi}(\zeta)|+\left|z^{\prime}\right|\right) .
$$

Finally, using that $|\zeta-z| \leq c|a(\zeta, z)|^{\frac{1}{2}}$ and $|\zeta-\Pi(z)|,\left|z^{\prime}\right| \leq c \mid a(\zeta, \Pi(z))^{\left.\right|^{\frac{1}{2}}}$ we obtain iii). 


\section{Lemma 4.2.}

If $\int$ is a function of class $L_{\delta, 0}^{p}(M)$, then the function $R_{M, \psi}^{s, r} f, \delta-1<s p$ is of class $L_{\delta^{*}, 0}^{p}(D)$ for all $\delta^{*} \geq \delta-l-(n+1+s+r) p, \delta^{*}>0$.

Proof:

Appliying the estimates of Theorem 2.4 of [3] and the same reasoning that in the Lemma 3.3 , we obtain the result.

\section{Corollary 4.3.}

If $f$ is a function of class $L_{\varepsilon, k}^{p}(M)$, then the function $R_{M, \psi}^{s, r} f, \delta-1<s p$ is of class $L_{\delta \cdot, k}^{p}(D)$ for all $\delta^{*} \geq \delta-l-(n+1+s+r) p ; \delta^{*}>0$.

\section{Proof:}

The proof is a consequence of the above Lemma and of the integration by parts formula given in the Iemma 2.5 .

\section{Lemma 4.4.}

Let be $f$ a $(0,1)$ form $\bar{\partial}$-closed with coefficients of class $L_{\delta, k}^{p}(D), \delta>p$ and let $u$ be a holomorphic function on a neighbourhood of $\bar{D}$, such thiat uf has coefficients of class $I_{\delta \cdot \frac{p}{2}, k}^{p}(D)$. Then there exists a function $g$ of class $L_{\delta-\frac{p}{2}, k}^{p}(D)$ such that $\bar{\partial} g=f$ and ug is of class $L_{\delta-p, k}^{p}(D)$.

\section{Proof:}

Wo take $g=-\int_{D} f \wedge K_{00}^{p}$ as in the Theorem 3.2 .

Hence, we only need to see that $u g$ is of class $L_{\delta-7, k}^{p}(D)$.

By (2.3) we have $\int_{D} g R_{00}^{s}=0$ and therefore we can write

$$
u(z) g(z)=\int_{D} u(\zeta) f(\zeta) \wedge K_{00}^{s}(\zeta, z)+\int_{D}(u(z)-u(\zeta)) g(\zeta) R_{00}^{s}(\zeta, z)
$$

The Theorem 3.2 gives that the first term is of class $L_{\delta-p, k}^{p}(D)$.

Moreover $(u(z)-u(\zeta)) R_{00}^{s}(\zeta, z)=R_{D, \psi}^{s_{1} \frac{1}{2}-(n+1+s)}$ and therefore by Corollary 4.3 we obtain that the second term is of class $L_{\delta-p, k}^{p}(D)$.

To prove the result of division given in the Theorem 1.1, first we consider the linear case to obtain local solutions. Finally using these solutions, the Lemma 4.4 and a result of division in the holomorplic Lipschitz spaces ([6]) we will obtain the result. 


\section{Proposition 4.5.}

If $Y=\left\{z ; z_{1}=0\right\}$ is transversal to the boundary of $D$, and $f$ is a function of closs $A_{\delta, k}^{p}(D)$ that is zero on $M$, then there exists a function $f_{1}$ of class $A_{\delta+\frac{p}{2}, k}^{p}(D)$ such that $f=z_{1} f_{1}$.

\section{Proof:}

We consider a covering $\left\{U_{i}\right\}_{i=0}^{i_{0}}$ of $D$ such that:

1) $U_{0}=\{z ; \rho(z)<-\delta<0\}$.

2) If $1 \leq i<i_{1}$ then $z_{1} \neq 0$ on $U_{i}$.

3) If $i_{1} \leq i \leq i_{0}$ then there exists a projection $\Pi_{i}$ as the one in the Lenma 4.1.

Let $\left\{\chi_{i}\right\}$ a partition of the unity for this covering.

We want to sce that $\chi_{i} \frac{f}{z_{l}}$ is a function of class $L_{\delta+\frac{p}{2}, k}^{p}(D)$.

Wc consider the three following cases.

1) $i=0$.

In this case using that $U_{0} \subset \subset D$ then we can take the function $\frac{f}{z_{1}}$ of class $C^{\infty}\left(\bar{U}_{0}\right)$ and therefore the result is true.

2) $1 \leq i<i_{1}$

In this case (4.1) is clear.

3) $i_{1} \leq i \leq i_{0}$

We will write $\Pi$ instead $\Pi_{i}$. Thus

$$
\begin{array}{r}
f(z)=f(z)-f(\Pi(z))=\int_{D} f(\zeta)\left(R^{s}(\zeta, z)-R^{s}(\zeta, \Pi(z))\right) d \zeta= \\
\int_{D} f(\zeta)\left(\frac{(-\rho(\zeta))^{s} \varphi(\zeta, z)}{a(\zeta, z)^{n+1+s}}-\frac{(-\rho(\zeta))^{s} \varphi(\zeta, \Pi(z))}{a(\zeta, \Pi(z))^{n+1+s}}\right) d \zeta
\end{array}
$$

where $\varphi(\zeta, z)$ is a function of class $\mathcal{C}^{\infty}(\bar{D} \times \bar{D})$ and holomorphic in $z$.

Using (4.2) $\mathrm{I}(z)-z=z_{1} h^{1}-d\left|z_{1}\right|^{2} \frac{\partial \rho}{\partial z^{\prime \prime}}$ where $h^{1}$ is a tangential complex vector, and thus we have

i) $\varphi(\zeta, z)-\varphi(\zeta, \Pi(z))=z_{1} \psi^{\prime}(\zeta, z)$ with $\psi^{\prime}(\zeta, z)$ of class $\mathcal{C}^{\infty}(\bar{D} \times \bar{D})$

ii) $a(\zeta, z)-a(\zeta, \Pi(z))=z_{1} \psi^{\prime \prime}(\zeta, z)$ with $\psi^{\prime \prime}(\zeta, z)$ of class $\mathcal{C}^{\infty}(\bar{D} \times \bar{D})$ and $\left|\Psi^{\prime \prime}(\zeta, z)\right|=O(|\zeta-z|+|\zeta-\Pi(z)|)$. (See (4.3)).

Hence, wo have

$$
\begin{aligned}
\chi_{i}(z) \frac{f(z)}{z_{j}} & =\int_{D} f(\zeta) \frac{(-\rho(\zeta))^{s} \chi_{i}(z) \psi(\zeta: z)}{a(\zeta, z)^{n+1+s}}+ \\
& \sum_{j=0}^{n+s} \int_{D} f(\zeta) \frac{(-\rho(\zeta))^{s} \chi_{i}(z) \psi_{1}(\zeta, z)}{a(\zeta, \Pi(z))^{n+1+s-j} a(\zeta, z)^{j+1}}
\end{aligned}
$$


where $\psi^{\prime}(\zeta, z), \psi_{1}(\zeta, z)$ are functions of class $\mathcal{C}^{\infty}(\bar{D} \times \bar{D})$ and $\psi_{1}(\zeta, z) \leq c(|\zeta-z|+|\zeta-\Pi(z)|)$.

With these notations we have that the above kernels are of the class $R_{D, \psi}^{s,-\left(n+\frac{3}{2}+s\right)}$ and therefore by Corollary 3.4 we obtain that $\chi_{i}(z) \frac{f(z)}{z_{1}}$ is a function of class $L_{\delta+\frac{\pi}{2}, k}^{p}(D)$.

Thus finally $f_{1}=\frac{f}{z_{1}}$ is of class $A_{\delta+\frac{p}{2}, k}^{p}(D)$.

\section{Definition 4.6.}

We say that the holomorphic submanifold $Y=\left\{z ; u_{1}(z)=\ldots=\right.$ $\left.u_{l}(z)=0\right\}$ is totally transversal to the boundary of $D$ if for every $1 \leq$ $j_{1}<\ldots<j_{s} \leq l, Y_{J}=\left\{z ; u_{j_{1}}(z)=\ldots=u_{j_{s}}(z)=0\right\}$ is a holomorphic submanifold of codimension $s$ and transversal to the boundary of $D$.

\section{Proposition 4.7.}

If $Y=\left\{z: z_{1}=\ldots=z_{i}=0\right\}$ is a holononphic submanifold totally transversal to the boundary of $D$ and $f$ is a function of class $A_{\delta_{3} k}^{p}(D)$ such that is zero on $M$, then there exist functions $f_{j}, j=1, \ldots, l$, of class $A_{\delta+\frac{p}{2}, k}^{p}(D)$ such that

$$
f=\sum_{j=1}^{\ell} z_{j} f_{j}
$$

Moreover, for all $j=1, \ldots$, the functions $z_{j} f_{j}$ are of class $\Lambda_{\delta, k}^{p}(D)$.

Proof:

We will construct the functions $f_{j}$ inductively.

Say $Y_{m}=\left\{z: z_{m+1}=\ldots=z_{t}=0\right\}, Y_{l}=C^{n}$ and $M_{m}=Y_{m} \cap D$.

Using the hypothesis of total transversavility we have that for each $m$, $M_{m}$ is a strictly pseudoconvex domain with boundary of class $\mathcal{C}^{\infty}$ and that $Y_{m-1}$ is transversal to the boundary of $M_{m}$.

By (1.1) we say that $\left.f\right|_{M_{1}}$ is a function of class $A_{\delta+i-1, k}^{p}\left(M_{1}\right)$ that is zero on $M_{0}$ and hence by Proposition 4.5 there exists a function $h_{1}$ of class $A_{\delta+l-1+\frac{p}{2}}^{p}\left(M_{1}\right)$ such that $f=z_{1} h_{1}$ on $M_{1}$.

We define $f_{1}(z)=\int_{M_{1}} R_{M_{1}}^{s}(\zeta ; z) h_{1}(\zeta) d \zeta$ where $R_{M_{\mathrm{l}}}^{s}$ is the extension operador (2.3).

By Lemma 4.2 we have that $f_{1}$ is of class $A_{\delta+\frac{p}{2}, k}^{p}(D)$.

Also putting

$$
z_{1} f_{1}(z)=\int_{M_{1}}\left(z_{1}-\zeta_{1}\right) f_{1}(\zeta) R_{M_{1}}^{s}(\zeta, z) d \zeta+\int_{M_{1}} f(\zeta) R_{M_{1}}^{s}(\zeta ; z) d \zeta
$$


and using that $\left|z_{1}-\zeta_{1}\right| \leq c|a(\zeta, z)|^{\frac{1}{2}}$ and the Corollary 4.3 we have that $z_{1} f_{1}$ is of class $A_{\delta, k i}^{p}(D)$.

If we consider the function $f-z_{1} f_{1}$ and we repent the above method on $M_{2}$ we will find $f_{2}$, and by iteration we will obtain the remaining $f_{j}$.

We introduce the following covering of $D$ which is a variation of the one of A.Cumenge [9].

\section{Lemma 4.8.}

For $0<\varepsilon_{1}<\ldots<\varepsilon_{r_{0}}$ there exist points $\left\{z_{i}\right\}_{i=1} \ldots, i_{0}$ of $D$ and strictly pseudoconvex domains with $\mathcal{C}^{\infty}$ boundary $\left\{D_{i}^{r}\right\}_{i=1, \ldots, i_{0}}^{r=1}$, such that:

i) $B\left(w_{i+} \varepsilon_{r-1}\right) \cap D \subset D_{i}^{r} \subset B\left(w_{i}, \varepsilon_{r}\right) \cap D$ if $1 \leq r \leq r_{0}$.

ii) $\cup_{i=1}^{i_{0}} D_{i}^{1}=D$.

iii) If $i_{1}<i \leq i_{0}$ there is $1 \leq i_{j} \leq l$ such that $u_{i_{j}} \neq 0$ in $D_{i}^{r}$.

iv) If $1 \leq i \leq i_{1}$ then

a) $D_{i}^{T} \cap Y \neq \emptyset$.

b) For every $D_{t}^{r_{0}}$ there exists a holomorphic system of coordinates such that the I first are $u_{1}, \ldots, u_{i}$.

v) $Y$ is totally transversal to $D_{i}^{r}$ for all $1 \leq i \leq i_{1}, 1 \leq r \leq r_{0}$.

vi) If $r<r^{\prime}$ and $D_{z_{1}}^{r} \cap \ldots \cap D_{i_{3}}^{r} \neq \emptyset$ then there exists a strictly pseudoconvex domain $D_{I}^{r}$ with $\mathrm{C}^{\infty}$ boundary, such that

(a) $D_{i, 1}^{r} \cap \ldots D_{i_{i}}^{r} \subset D_{l}^{r} \subset D_{i_{1}}^{r^{r}} \cap \ldots \cap D_{i_{s}}^{r^{\prime}}$.

b) If $D_{I}^{r} \cap Y \neq 0$ then $Y$ is totally transversal to the boundory of $D_{I}^{r}$.

Proof of Theorem 1.1:

We take the covering of $D$ of the Lemma 4.8. We fix an $r$ and we write $D_{i}$ instead $D_{i}^{r}$.

By Proposition 4.7 in each $D_{i}$ we lave:

$$
\begin{aligned}
& f(z)=\sum_{j=1}^{l} n_{j}(z) f_{j}^{i}(z) \\
& f_{j}^{i}(z) \in A_{\delta+\frac{p^{2}: k}{p}}^{p}\left(D_{i}\right), \quad u_{j} f_{j}^{i} \in A_{\delta, k}^{p}\left(D_{i}\right)
\end{aligned}
$$

We define $g_{j}(z)=\sum_{i} \chi_{i}(z) f_{j}^{i}(z)$ where $\left\{\chi_{i}\right\}$ is a partition of the unity wilh respect to the covering $\left\{D_{i}\right\}$.

It is clear that $\sum_{j=1}^{l} u_{j} g_{j}=f$. 
For each $j$ we denote by $w_{j}$ the solution of the equation $\bar{\partial} w_{j}=\bar{\partial} g_{j}$ given by the Lemma 4.3 and we put

$$
f=\sum_{j=1}^{l} u_{j}\left(g_{j}-w_{j}\right)+\sum_{j=1}^{l} u_{j} w_{j}
$$

By Lemma 4.4 and (1.2) we have

$$
\begin{aligned}
h_{j} & =g_{j}-w_{j} \in A_{\delta+\frac{p}{2}, k}^{p}(D) \\
h & =\sum_{j=1}^{l} u_{j} w_{j} \in A_{\delta+\frac{p}{2}, k+1}^{p}(D) .
\end{aligned}
$$

Hence, we have proved that for every function $\int \in A_{\delta, k}^{p}(D)$ that is zero on $M$, there exist functions $h_{y} \in A_{\delta+\frac{p}{2}, k}^{p}(D)$ and $h \in A_{\delta+\frac{p}{2}, k+1}^{p}(D)$ such that

i) $f=\sum_{j=1}^{l} u_{j} h_{j}+h$

ii) $h$ is zero on $M$.

Iterating this method with the function $h$ we obtain

i) $f=\sum_{j=1}^{l} u_{j} h_{j}^{r}+h^{r}$

ii) $h^{r} \in A_{\delta+\frac{r p}{2}, k+r}^{p}(D)$ and is zero on $M$

iii) $h_{j}^{r} \in A_{\delta+\frac{p}{2}, k}^{p}(D) \quad j=1, \ldots, l$.

Taking $r$ such that $t=k-\frac{n+\delta}{p}+\frac{r}{2}>k+\frac{1}{2}$ and applying (1.1) we have that $h^{r}$ is a holomorphic Lipschilz function of class $A^{l}(D)$ that is zero on $M$. Therefore by a result of [6] we have

$$
h^{r}=\sum_{j=1}^{l} u_{j} h_{j}^{r+1}, \quad h_{j}^{r+1} \in A^{i-\frac{1}{2}}(D) \subset \mathcal{C}^{k}(\bar{D}) \cap \mathcal{O}(D) .
$$

Finally, if we define $f_{j}=h_{j}^{r}+h_{j}^{r+1}$ we end the proof.

\section{Extension of $A_{\delta, k}^{p}$-jets}

First we prove the extesion rcsult in the linear case.

Theorem 5.1.

If the linear submanifold $Y=\left\{z \in C^{n} ; z_{1}=\ldots=z_{l}=0\right\}$ is transversal to the boundary of $D$ and $F$ is an $A_{\delta, k}^{p}$-jet of order $m$ on $M$ 
then there exists a fanction $f$ of class $A_{\delta, k}^{p}(D)$ such that $J_{m} f=F$ on M.

Proof:

First we consider the case $Y=\left\{z ; z_{1}=0\right\}$.

We take $s>\frac{\delta}{y}$ and for $j=0, \ldots, m$ we define by induction the functions

$$
g_{0}=E^{s} F^{0}
$$

$$
g_{j}=g_{j-1}+\frac{z_{1}^{j}}{j !} E^{s}\left(F^{j}-d^{j} g_{j-1}\right)\left(\frac{\partial}{\partial \zeta_{1}} \ldots, \frac{\partial}{\partial \zeta_{1}}\right)
$$

where the operator $E^{s}$ is the extension operator (2.4) given by the kernel $R_{M}^{s}$.

It is clear that the function $f=g_{m}$ satisfies $J_{n} f=F$ on $M$.

To prove the Theorem we will show by induction on the index $j$ in (5.1) that the functions $g_{j}$ are of class $A_{\delta, k}^{p}(D)$.

If $j=0$, using that $R_{M}^{s}=R_{M, \psi}^{s,-(n+1+s)}$ and applying the Corolary 4.3, we obtain the result.

Now we assume that $g_{j-1} \in A_{\delta, k}^{p}(D)$. As follows from (5.1), to prove that $g_{j} \in A_{\delta, k}^{p}(D)$ is sufficient to see that

$$
h_{j}=z_{1}^{j} \int_{M} R_{M}^{s}\left(F^{j}-d^{j} g_{j-1}\right)\left(\frac{\partial}{\partial \zeta_{1}}, \ldots: \frac{\partial}{\partial \zeta_{1}}\right)
$$

is of class $A_{\delta, k}^{p}(D)$.

Consider the normal complex field

$$
N=\frac{1}{\mid \partial \rho_{i}^{2}} \sum_{i=1}^{n} \frac{\partial \rho}{\partial \bar{\zeta}_{i}} \frac{\partial}{\partial \zeta_{i}}
$$

defined in a neighbourhood of the boundary of $D$, and the decomposition of the vector field

$$
Z=\sum_{i=1}^{n}\left(z_{i}-\zeta_{i}\right) \frac{\partial}{\partial \zeta_{i}}=\sum_{i=1}^{n}\left(z_{i}-\zeta_{i}\right)\left(\frac{\partial}{\partial \zeta_{i}}-\chi \frac{\partial \rho}{\partial \zeta_{i}} N\right)+\chi Z \rho N
$$

where $\chi$ is a function with compact support and that is 1 in a neighbourhood of the boundary of $D$. 
We denote by $T_{i}$ the complex tangent vector field $T_{i}=\frac{\partial}{\partial \zeta_{i}}-\frac{\partial \rho}{\partial \zeta_{i}} N$.

With these notations and by the properties I-1, I-2 and I-3 of the Definition 1.1, we can write

$$
\begin{gathered}
h_{j}=\int_{M} R_{M}^{p}\left(F^{j}-d^{j} g_{j-1}\right)(z-\zeta, \ldots, z-\zeta)= \\
\sum_{|\beta|=j} \int_{M} R_{M}^{p}\left(z_{1}-\zeta_{1}\right)^{\beta_{1}} \ldots\left(z_{n}-\zeta_{n}\right)^{\beta_{2}}(Z \rho)^{\beta_{n+1}} g_{\beta}
\end{gathered}
$$

where $g_{\beta}=\left(F^{j}-d^{j} g_{j-1}\right)\left(T_{1}, . .{ }^{\left(\beta_{1}\right)} . ., T_{1}, \ldots, N, . .{ }^{\left(\beta_{n+1}\right)}{ }_{. .}, N\right)$.

Observe that by the hypothesis of induction and the property I-4, we have that the function $g_{\beta}$ is of class $L_{\delta+\frac{\beta_{1}+\ldots+\beta_{n}}{2}}^{p}+\beta_{n+1}, k(M)$.

Moreover, using that $|\zeta-z|^{2},|Z \rho| \leq c|a(\zeta, z)|$ we can write

$$
h_{j}=\sum_{|\beta|=j} R_{M, \psi_{\beta}}^{s, r_{3}-\langle n+1-i+s\}} g_{\beta}: \quad r_{\beta}=\frac{\beta_{1}+\ldots+\beta_{n}}{2}+\beta_{n+1}
$$

and applying the Corollary 4.3 we end the proof in this case.

The proof in the case $Y=\left\{z ; z_{1}=\ldots=z_{l}=0\right\}$ is similar to the case $Y=\left\{z_{i} z_{1}=0\right\}$. In the same way, in this case the function $f$ is defined by $f=g_{m}$, where

$$
\begin{aligned}
& g_{0}=E^{p} F^{0} \\
& g_{j}=g_{j-1}+E^{p}\left(\left(F^{j}-d^{j} g_{j-1}\right)(z-\zeta, \ldots, z-\zeta)\right) .
\end{aligned}
$$

Before proving the Theorem 1.3 we introduce the following definition.

\section{Definition 5.2.}

For every $\varepsilon \geq 0$ small enough, we define

$$
D_{\varepsilon}=\left\{\zeta ; \rho(\zeta)-\varepsilon|u(\zeta)|^{2}<0\right\}
$$

where $|u|^{2}=\left|u_{1}\right|^{2}+\ldots+\left|u_{l}\right|^{2}$.

It is clear that these domains are strictly psendoconvex domains with $\mathcal{C}^{\infty}$ boundary, $D_{\varepsilon} \cap Y=M$ and $Y$ is transversal to $D_{\varepsilon}$.

\section{Lemma 5.2.}

If $f \in L_{\delta, k}^{p}\left(D_{\varepsilon^{\prime}}\right), \delta>\frac{p}{2}$, then $u_{j} f \in L_{\delta-\frac{p}{2}, k}^{p}\left(D_{\epsilon}\right)$ for every $j=$ $1, \ldots, l$, and $0 \leq \varepsilon<\varepsilon^{\prime}$. 
Proof:

The result is a consequence of the fact that

$$
\left|u_{j}\right| \leq \frac{1}{\left(\varepsilon^{\prime}-\varepsilon\right)^{\frac{1}{2}}}\left(-\rho+\varepsilon^{\prime}|u|^{2}\right)^{\frac{1}{2}} ; \quad \text { on } D_{\varepsilon}
$$

for all $\delta^{*} \geq \delta-p, \delta^{*}>0$

Proof of Theorem 1.3:

We take a covering $\left\{D_{i}^{r}\right\}=\left\{D_{i}^{r}\right\}_{i=1 ; \ldots, N}^{0 \leq r \leq r_{0}}$ of $D$ as the one in the Lemma 4.8 and we also consider the domains $\left\{D_{i, \varepsilon}^{r}\right\}, \varepsilon \geq 0$.

We also take $0<r<r^{\prime \prime}<r^{\prime}, 0<\varepsilon<\varepsilon^{\prime}$.

By Proposition 5.1 we have that for every $D_{i, \varepsilon^{\prime}}^{r^{\prime}}$ such that $D_{i} \cap Y \neq \emptyset$, there exists a function $f_{i} \in A_{\delta, k}^{y}\left(D_{i, \varepsilon^{\prime}}^{r^{\prime}}\right)$ such that $J_{m n} f_{i}=F$ on $Y \cap D_{i}$.

Using (1.2) we can assume that $\delta>p$.

For the remaining $D_{i}$ we define $f_{i}=0$.

We consider the function $g=\sum_{i} \chi_{i} f_{i}$ where $\chi_{i}$ is a partition of the unity with respect to the $\left\{D_{i . \epsilon^{\prime}}^{r^{\prime}}\right\}$.

This function $g$ is of class $L_{\delta, k}^{p}(D)$ and verifies $J_{m_{t}} g=F$.

Let $w \in L_{\delta-\frac{p}{2}, k}^{p}(D)$ be the solution of the $\bar{\partial} w=\bar{\partial} g$ given by Lemma 4.4 .

Note that $h=g-w \in A_{\delta, k}^{p}(D)$ and that $F=J_{m} h+J_{m} w$.

The next step is to sce that $J_{m} w$ is an $A_{\delta+\frac{p}{2}, k+1^{-}}^{\text {-jet. }}$

We say $f_{i j}=f_{i}-f_{j}$ in $D_{i j}^{r^{\prime \prime}} \subset D_{i}^{r^{\prime}} \cap D_{j}^{r^{\prime}}$.

Using the Theorema 1.1 we can write

$$
f_{i j}=\sum_{|\gamma|=n+1} u^{\gamma} g_{i j}^{\gamma}, \quad g_{i j}^{\gamma} \in A_{\delta+\frac{(m+1) p}{2}, k}^{p}\left(D_{i j, \varepsilon^{\prime}}^{r^{\prime \prime}}\right) .
$$

We define in $D_{i}^{r}$ the function $g_{i}^{\gamma}=\sum_{s} \chi_{s} g_{i s}^{\gamma}$.

This function satisfies

$$
\sum_{|\gamma|=n+1} u^{\gamma} g_{i}^{\gamma}=f_{i}-\sum_{s} \chi_{s} f_{s}=f_{i}-g .
$$

By Lomma 4.4 we can take $w_{i}^{\gamma}$ such that;

$$
\bar{\partial} w_{i}^{\gamma}=\bar{\partial} g_{i}^{\gamma} \quad, \quad w_{i}^{\gamma} \in L_{\delta+\frac{m p}{2}, k}^{p}\left(D_{i, \varepsilon^{\prime}}^{\gamma^{\prime \prime}}\right)
$$


Moreover; using the Lemma 4.4, the Lemma 5.2 and (1.2) we have that

$$
h_{i}^{\prime}=w-\sum_{|\gamma|=n_{n+1}} u^{\gamma} w_{i}^{\gamma} \in A_{\delta+\frac{p}{2}, k+1,1}^{p}\left(D_{i, \epsilon}^{r}\right)
$$

and also $J_{m} h_{i}^{\prime}=J_{m} g$ on $Y \cap D_{i}^{r}$.

Hence, we have that $J_{m} g$ is a $A_{\delta+\frac{p}{2}, k+1}^{p}$-jet of order $m$ on $M$.

By iteration of this method we obtain

$$
F=J_{m} h^{s}+J_{m} g^{s}
$$

with

$$
h^{s} \in A_{\delta_{1} k}^{p}(D) \quad \text { and } \quad J_{m n} g^{s} \text { is in } A_{\delta+\frac{s p}{2}, k+s^{-j e t}}^{p}
$$

Now if we take $s$ such that $t=k+s-\frac{n+\delta}{p}-\frac{s}{2}>k+\frac{1}{2}$, then (I.1), (1.4) and (1.6) shows that $J_{m} g^{s}$ is a $A^{t}$-jet of order $m$. Finally applying the extension result of $A^{t}$-jets (1.6) we can take a function $h$ of class $A^{t}(D)$ such that $J_{m n} h=J_{m} g^{s}$ on $M$ and defining $f=h^{s}+h$ we end the proof.

\section{References}

1. Ahern, P., Bruna, J., Maximal and arca integral characterizations of Hardy-Sobolev spaces in the unit ball of $C^{n}$, Rev. Mat. Iberoamericana 4 (1988), 123-153.

2. Beatrous, F., $L^{p}$ estimates for extensions of holomorphic functions, Michigan Math. J. 32 (1985), 361-380.

3. BEATROUS, F., Estimates for derivatives of holomorphic functions in pseudoconvex domains, Math. Z. 191 (1986), 91-116.

4. Beatrous, F., Burbea, J., Sobolev spaces of holomorphic functions in the ball, Dissertationes Math. 276 (1989).

5. Berndtsson, B., Andersson, M., Henkin-Ramirez Formulas with weight factors, Ann. Inst. Fourier (Grenoble) 32 (1983), 91-110.

6. Bonneau, P., Cumenge, A., Zériahi, A., Division dans les espaces de Lipschitz de fonctions holomorphes, C. R. Acad. Sc. Paris 297 (1983), 517-520.

7. BRUNA, I., BURGUÉs, J. M., Holomorphic approximation in $C^{m}$-norms on totally real compact sets in $C^{n}$, Math. Ann. 269 (1984), 103-117. 
8. BrunA, J., ORTEGA, J. M., Traces on curves of Sobolev spaces of holomorphic functions, Arkiv Mot. 29 (1991), 25-49.

9. Cumenge, A, Exlension dans les classes de Hardy de fonctions holomorphes et estimations de type "Mesures de Carleson" pour l'equation $\bar{\partial}$, Annules de l'Institut Fourier 33, 3 (1983), 59-97.

10. Dautov, S. A., Henkin, G. M., The zeroes of holomorphic functions of finite order and weight estimates for solutions of the J̈-equation, Math. USSR. Sbornik 35 (1979), 449-459.

11. ORTEGA, J. M., The Gleason problern in Bergman-Sobolev spaces, to appear in Complex Variables.

12. Ortega, J. M., FàbreGA, J., Extension of $A^{t}$-jets from holomorphic submanifolds, to appear in Math. Z.

Departament de Matemàtica Aplicada i Anàdisi

Facultal de Matemètiques

Universitat de Barcelona

Gran Via de les Corts Calatanes 585

08007 Barcelona

SPAIN

Rebut el 18 de Desembre de 1991 